\title{
Magnetic resonance imaging-navigated argon-helium cryoablation therapy against a rabbit VX2 brain tumor
}

\author{
YANG WANG ${ }^{1}$, HONG-LI KAN ${ }^{2}$, HONG SUN ${ }^{2}$, DONG-XIN WANG ${ }^{2}$, HUAI-WU WANG ${ }^{2}$ and JI-XIN LIU ${ }^{2}$ \\ ${ }^{1}$ Department of Interventional Oncology, Jilin Provincial People's Hospital, Changchun, Jilin 130021; \\ ${ }^{2}$ Department of Anesthesia, Tumor Hospital of Jilin Province, Changchun, Jilin 130012, P.R. China
}

Received May 5, 2014; Accepted December 11, 2014

DOI: $10.3892 /$ etm.2015.2375

\begin{abstract}
The aim of the present study was to investigate the feasibility of interventional magnetic resonance imaging (MRI)-guided and monitored argon-helium cryoablation for the treatment of brain tumors in rabbits. In addition, the present study evaluated the associations between imaging and pathology, the therapeutic effects and the effects on the surrounding normal tissues. A total of 14 rabbits were equally divided into groups $\mathrm{C}$ and D. Under general anesthesia, the skull was drilled and tumor blocks were implanted. Subsequently, a New Zealand rabbit VX2 brain tumor model was successfully established. Rabbits in group $\mathrm{C}$ were treated with argon-helium cryoablation and those in group D did not undergo any treatment (control). Regular postoperative MRI scanning was performed to observe the changes in tumor size, and the survival times of the rabbits in groups $C$ and $D$ were recorded. The extent of necrosis in the brain tumor exhibited a significant correlation with the freezing time of cryoablation, and the necrotic region was shown to be the same size as the ice ball. The survival times of the rabbits in the treatment group (group C) were significantly prolonged. Therefore, the observations of the present study demonstrated that the VX2 brain tumor model, produced using an improved tumor block implantation method, was stable and suitable for MRI observation and interventional study. In addition, argon-helium cryoablation was shown to be a safe and feasible therapeutic method for the treatment of brain tumors, and was demonstrated to significantly increase the survival times of the brain tumor-bearing rabbits.
\end{abstract}

Correspondence to: Dr Hong-Li Kan, Department of Anesthesia, Tumor Hospital of Jilin Province, 1,018 Huguang Road, Chaoyang, Changchun, Jilin 130012, P.R. China

E-mail: hnoglikan@163.com

Key words: magnetic resonance imaging intervention, rabbit, animal model, brain tumor, cryoablation

\section{Introduction}

Primary brain tumors have a high incidence rate, and tumor growth is invasive with an obscure boundary (1). Although brain tumors can be resected through various treatment methods, including surgery, radiotherapy and chemotherapy, disease progression is unable to be effectively controlled. In addition, the prognosis of patients with a brain tumor is usually poor, with a short survival time and severe sequelae (2).

Magnetic resonance imaging (MRI)-navigated argonhelium cryoablation is a new advanced technology. MRI is known to have good tissue resolution and a multiplanar imaging capability. In particular, the temperature-sensitive characteristics of MRI, which enable the clear and real-time display of the size and range of the ice ball (which destroys the tumor cells), has resulted in this imaging technique becoming the focus in clinical cryotherapy against multiple-system tumors, with satisfactory results previously achieved (3-7). Although MRI has an unparalleled advantage in nervous system imaging compared with other methods (8), there are a limited number of domestic and foreign studies investigating MRI-navigated cryotherapy for the treatment of brain tumors.

In the present study, a rabbit VX2 brain tumor model was established that was suitable for MRI imaging and interventional therapy. The model was used for the preliminary study of MRI-navigated argon-helium cryoablation therapy against the brain tumor.

\section{Materials and methods}

Animals. In total, 24 New Zealand white rabbits were provided by the Department of Experimental Animals at the Quality Control Center of Shandong Lukang Pharmaceutical Group Co., Ltd. (Jining, China). The rabbits were male or female and weighed between 2.2 and $3.4 \mathrm{~kg}$. A total of 2 of the rabbits succumbed in normal captivity for unexplained reasons. The study was conducted in strict accordance with the recommendations in the Guide for the Care and Use of Laboratory Animals of the National Institutes of Health. In addition, the animal use protocol was reviewed and approved by the Institutional Animal Care and Use Committee of Jilin Provincial People's Hospital (Jilin, China). 
Tumor cells. Following resuscitation, the VX2 tumor cells (presented by Professor Chengli Li of the Shandong Medical Imaging Research Institute, Jinan, China) were conventionally adherence-cultivated in RPMI 1640 complete medium, which contained $20 \%$ fetal bovine serum, $50 \mathrm{IU} / \mathrm{ml}$ penicillin, $50 \mu \mathrm{g} / \mathrm{ml}$ streptomycin and $300 \mu \mathrm{g} / \mathrm{ml}$ glutamine. Next, logarithmically-growing VX2 tumor cells were obtained for digestion with $0.25 \%$ trypsin. The cell samples were subsequently centrifuged at $377 \mathrm{x}$ g for $5 \mathrm{~min}$, after which the supernatant was discarded. Following washing with Dulbecco's modifed Eagle's medium (DMEM), the precipitate was diluted with DMEM to form a cell suspension liquid with a concentration of $1 \times 10^{6}$. Cell viability was assessed using a trypan blue assay, and the survival rate of the inoculated tumor cells was required to be $>90 \%$. A $1-\mathrm{ml}$ syringe was used to transfer $0.5 \mathrm{ml}$ cell suspension liquid into the rabbit bilateral posterior thigh muscle for subcultivation.

Establishment of the brain tumor model. Anesthesia was induced using $20 \mathrm{mg}$ ketamine and $0.5 \mathrm{mg}$ midazolam via intramuscular injection (9).

Acquisition of tumor tissue was performed as follows. The rabbit VX2 solid tumor in the hind legs was resected under sterile surgical conditions, the outer layer of fat was removed and the blood was washed away with Hank's solution. Next, the solid tumor was cut open and the inner rotten porridge-like necrotic tissues were removed to leave the red-white meat-like fresh tissue that had a greater peripheral vascular component and grew vigorously. The tissue was subsequently cut into $1.5-\mathrm{mm}$ sections with a scalpel, while a sterile gelatin sponge was cut into $1.5 \times 3 \mathrm{~mm}$-length strips. The tumor blocks and gelatin sponge strips were then implanted into a $18 \mathrm{G}$ sterile needle (Suzhou Antai Medical Devices Co., Ltd., Suzhou, China) for future use.

In the cultivation method, the rabbit was firstly anesthetized and fixed in the prone position. Following the removal of top hair and disinfection, a $2-3 \mathrm{~cm}$ incision was made on the lateral canthus of the eyes and upwards along the median sagittal plane. The periosteum was subsequently separated and a $16 \mathrm{G}$ bone driller (Shenyang Pusiman Medical Instrument Co., Ltd., Shenyang, China) was applied at a distance $5 \mathrm{~mm}$ to the right of the sagittal suture and $5 \mathrm{~mm}$ after the coronal suture. The aforementioned tumor block prepared in the $18 \mathrm{G}$ puncture needle was punctured through the hole in the bone into a depth of $\sim 7 \mathrm{~mm}$. The needle was fixed, and the needle core was used to implant the tumor block and the gelatin sponge into the right frontal lobe. The needle was slowly withdrawn and the hole in the bone was sealed with bone wax. Finally, the scalp was sutured.

Grouping, treatment and observation. The brain tumor model was prepared in two stages. In the first stage, eight rabbits received the brain tumor block implantation and were subsequently divided into groups A and B, with four rabbits in each group. Under the guidance of an interventional MRI System $(10,11)(0.3$ T open MRI scanner with iPath200 optical tracking guidance system and foldable multifunctional coil; XinAoMDT Technology Co., Ltd., Langfang, China), cryoablation using a CRYO-HITTM argon-helium knife with MRI compatibility (7) (1.47 mm probe; Galil Medical, Inc.,
Jerusalem, Israel) was performed once the tumor diameter had reached $\sim 0.8 \mathrm{~cm}(\sim 15$ days after the tumor tissue implantation). In group A, two cycles of argon-helium freezing-heating ablation was performed, with 5 min freezing and then heating to $40^{\circ} \mathrm{C}$ in each cycle (12). The group B rabbits also underwent two cycles of freezing-heating ablation; however, $10 \mathrm{~min}$ freezing and then heating to $40^{\circ} \mathrm{C}$ was applied in each cycle. The second stage of tumor model preparation was performed according to the optimal ablation conditions determined from the first stage. In the second stage, a total of 14 rabbits underwent brain tumor implantation, which were divided into groups $\mathrm{C}$ and D, with seven rabbits in each group. MRI scanning was performed 10 days after the tumor block implantation, followed by five-day interval scanning until the tumor grew to a diameter of $0.8 \mathrm{~cm}$. Subsequently, the rabbits in group $\mathrm{C}$ underwent argon-helium cryoablation, with the conditions decided under the optimal condition of the first stage (group B). Postoperative MRI scanning was performed once a week. The group D rabbits were used as controls. They did not receive any treatment and only underwent MRI scanning once every five days. The tumor volume $\left(\mathrm{mm}^{3}\right)$ was calculated according to the following formula (13): $\mathrm{V}=(4 / 3 \times \pi \times \mathrm{L} \times \mathrm{W} \times \mathrm{H}) \times 1 / 8$, where $\mathrm{L}, \mathrm{W}$ and $\mathrm{H}$ were the maximum anteroposterior diameter, width and height, respectively, of the tumor, as measured by enhanced MRI. Routine enhanced MRI scanning was performed prior to the sacrifice of the rabbits, after which the tumor specimens were obtained.

MRI-navigated argon-helium cryoablation therapy for the $V X 2$ brain tumor. MRI scanning was initiated at day 10 after the implantation of the VX2 tumor blocks into the New Zealand rabbits, followed by three-day interval scanning. A total of $0.8 \mathrm{ml} / \mathrm{kg}$ gadolinium $(\mathrm{Gd})$-diethylene triamine pentaacetic acid (DTPA; Bayer Schering Pharma AG, Berlin, Germany) was injected through the ear vein for further observation. When the tumor grew to a diameter of $\sim 0.8 \mathrm{~cm}$, MRI-navigated argon-helium cryoablation was performed. The top hair of the rabbits was removed again, after which the rabbits were anesthetized and fixed on a special-crafted fixer for MRI interventional pre-scanning. In the pre-scanning images, the tumor center was set as the target. Following disinfection and sterile sheet paving, the scalp was re-cut along the last surgical incision and the periosteum was separated. The argon-helium probe was inserted through the tumor block implantation hole for freezing-heating ablation. Intraoperative real-time scanning was performed to monitor the entire ablation process.

Histopathological examination. Group A and B rabbits underwent MRI scanning at the following time points: Immediately following ablation and at days 7 and 14 after treatment. After each scanning procedure, one rabbit from each group underwent left ventricular perfusion with $4 \%$ paraformaldehyde. The rabbit was dissected and the brain tissue was fixed in $4 \%$ formaldehyde solution. After one day, the brain was cut into 2-3-mm continuous coronal slices for general observations of the lesion size, shape and edge following ablation. The typical slice was subsequently embedded in paraffin and stained with hematoxylin and eosin (HE) to observe the histological changes of the ablation zone. A similar method was performed 
Table I. Association between the tumor lesion size $\left(\mathrm{mm}^{3}\right)$ and the time point of magnetic resonance imaging scanning in the group D rabbits.

\begin{tabular}{lcccccc}
\hline Rabbit & Day 10 & Day 15 & Day 20 & Day 25 & Day 30 & Day 35 \\
\hline No. 1 & 39.56 & 298.68 & 991.85 & 2755.18 & 5326.40 & 9731.16 \\
No. 2 & 57.48 & 405.70 & 1972.54 & 5902.11 & & \\
No. 3 & 44.83 & 349.74 & 1545.77 & 4217.93 & 7485.54 & 14038.98 \\
No. 4 & 45.68 & 320.14 & 1236.81 & 3704.25 & 5993.47 & \\
No.5 & 47.37 & 450.79 & 2200.32 & 6418.57 & & \\
No. 6 & 40.94 & 302.15 & 1243.08 & 4767.54 & 8994.66 & 15249.82 \\
No. 7 & 45.35 & 311.43 & 1194.93 & 4438.19 & 9447.08 & \\
\hline
\end{tabular}

to the rabbits in group $\mathrm{D}$ for the collection of general specimens and the pathological examination of the moribund rabbits. The tumor volume was calculated according to the following formula: $\mathrm{V}=\mathrm{a}^{2} \mathrm{x} \mathrm{b} / 2$, in which a represented the long tumor diameter and $b$ represented the short tumor diameter, with the unit set as $\mathrm{mm}^{3}$.

Statistical analysis. SPSS 16.0 statistical software (SPSS, Inc., Chicago, IL, USA) was used for statistical analysis. The counting data were analyzed using the t-test, while survival analysis was conducted using Kaplan-Meier survival curves. The log-rank test was used to assess survival distribution between groups $\mathrm{C}$ and $\mathrm{D}$, while correlation analysis was performed using Pearson's method. $\mathrm{P}<0.05$ was considered to indicate a statistically significant difference.

\section{Results}

Situation of the tumor implantation. Among the eight New Zealand white rabbits that received the tumor block implantation during the first stage, one rabbit in group A exhibited a brain abscess caused by postoperative intracranial infection (MRI scan revealed a ring-like enhancement lesion and the pathological examination confirmed the abscess). An additional rabbit in group $\mathrm{B}$ died as a result of contrast agent pollution-induced sepsis during the MRI scanning. Thus, there were six remaining experimental animals. In the second stage of tumor model assessment, MRI scanning revealed that a tumor had grown in the 14 rabbits. The overall rate of tumorigenicity was $90.91 \%$, and the follow-up MRI scanning revealed that none of the tumors exhibited spontaneous shrinkage or disappearance.

MRI results. At day 10 following the tumor block implantation, the lesions were observed to be round or oval, with a maximum average diameter of $4.4 \pm 1.1 \mathrm{~mm}$. The T1-weighted images (WIs) exhibited a slightly lower or equal signal, while the T2WI and fluid-attenuated inversion recovery (FLAIR) scans exhibited a slightly hyperintense signal with clear edges and without evident peripheral edema. Following an injection of gadolinium (Gd)-DTPA, the scanning results revealed that the tumor exhibited marked homogeneous enhancement. When the tumor was $>1.2 \mathrm{~cm}$, the lesion center exhibited necrosis, and the T1WI and T2WI revealed a centered low signal. The association between the tumor lesion size and the various time points of MRI scanning in the rabbits from group D was analyzed, as shown in Table I and Fig. 1.

During MRI-navigated and monitored cryoablation, the ice ball was exhibited as an oval-shaped signal deletion region with a clear boundary in all the imaging scans. Following cryoablation, the group B rabbits exhibited more typical MRI scan evolutions. On postoperative day 7 , the ablation zone in the T1WI exhibited an equal signal, with a diameter slightly larger compared with the previous measurement, which was surrounded by a ring-like enhancement. The ablation zone in the T2WI exhibited an inhomogeneous hyperintense signal, which was surrounded by an incomplete hypointensity hemosiderin ring. The outer edge of the hemosiderin ring exhibited irregular flaky edema. At day 14 after the ablation, the ablation zone in the T1WI continued to show an equal signal surrounded by a homogeneous enhancement ring, while the diameter had decreased to almost to the size of the ice ball. The ablation zone in the T2WI revealed a homogeneous hyperintense signal, surrounded by a uniform-thickness isointensity ring, and the peripheral edema had almost disappeared. The ablation zones in the T1WI and T2WI from the rabbits in group A on postoperative days 7 and 14 all showed heterogeneous signals, while the peripheral ring shadow was irregular and continuous.

General specimen and pathological results. General morphological observations revealed the rabbit brain VX2 tumors were round, without an envelope and a slightly blurred boundary contour. In addition, the incisal surface was pale red to dark red. The HE staining results revealed that the tumor cells grew actively, were arranged densely and were palisade or coral-like shaped. The boundary between the tumor and normal tissues exhibited infiltration of tumor cells towards the adjacent brain parenchyma; therefore, the boundary exhibited a certain degree of blurring. The blood vessels were rich within the tumor, with small flaky necrosis observed occasionally in the center. The tumor volumes calculated from the MRI scanning of the group D rabbits are shown in Table II.

The data revealed that the volumes calculated from the general specimen measurement were slightly less compared with volume calculated from MRI scanning. However, the paired t-test revealed that there was no statistically significant difference between the two measurement protocols $(t=4.094$; $\mathrm{P}=0.006)$. Pearson's correlation analysis revealed that the aforementioned two exhibited a significant correlation $(r=0.99$; $\mathrm{P}<0.01)$. 
Table II. Tumor volume calculations $\left(\mathrm{mm}^{3}\right)$ from MRI scanning and the general pathological volume of the tumors in the group D rabbits.

\begin{tabular}{lcccccc}
\hline Measurement volume & No. 1 & No.2 & No.3 & No.4 & No. 5 & No.6 \\
\hline MRI & 11045.7 & 6532.3 & 8420.7 & 14039.0 & 6419.0 & 10194.3 \\
General specimen & 10011.4 & 5896.9 & 7537.3 & 12430.8 & 5792.4 & 8978.6 \\
\hline
\end{tabular}

MRI, magnetic resonance imaging.

Table III. Comparison of the survival times of the rabbits in groups C and D (days).

\begin{tabular}{lccccccc}
\hline Group & No. & No. 2 & No. 3 & No. & No.5 & No.6 & No.7 \\
\hline C & $60^{\mathrm{a}}$ & 53 & $60^{\mathrm{a}}$ & 39 & 46 & $60^{\mathrm{a}}$ & 33 \\
$\mathrm{D}$ & 39 & 28 & 33 & 36 & 29 & 32 \\
\hline
\end{tabular}

${ }^{\mathrm{a} C e n s o r e d ~ v a l u e s . ~}$

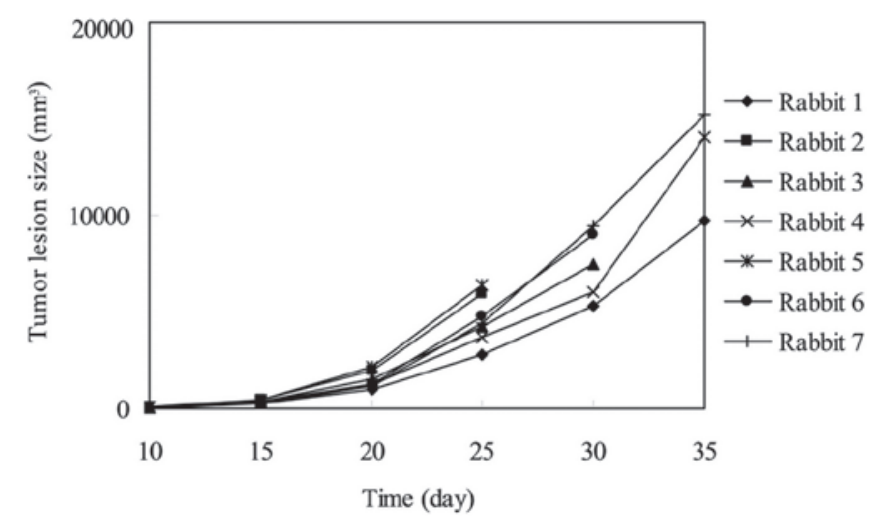

Figure 1. Tumor lesion size-time curve in the group D (control) rabbits. These rats did not receive any treatment and thus the normal tumor growth in the body is shown.

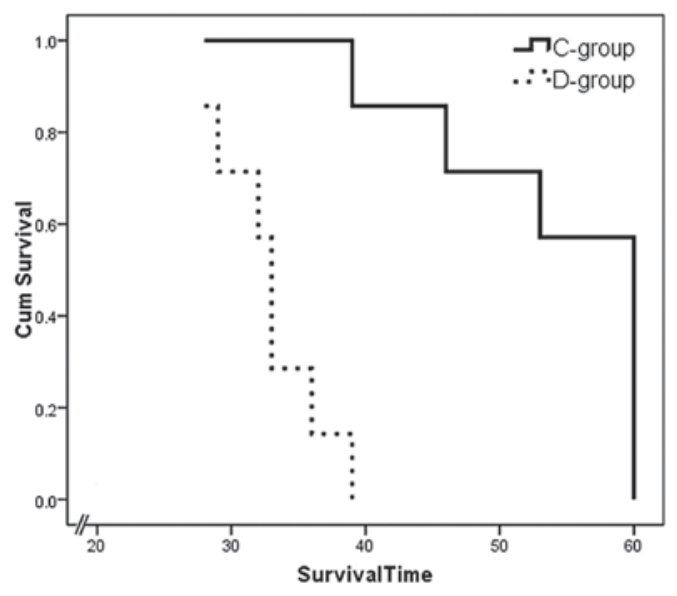

Figure 2. Survival function of the rabbits in groups $\mathrm{C}$ and $\mathrm{D}$.

Brain samples from the rabbits, obtained from the immediate sacrifice following cryoablation, revealed that the ablation zone was clear, with a sharp dark brown edge. In addition, pathological examination revealed that the tumor cells in the brain specimens from the group A rabbits exhibited slight swelling when compared with the surrounding normal nerve cells; however, the changes were not significant. The majority of tumor cells in the group B rabbits were markedly swollen, and a few tumor cells had ruptured. On days 7 and 14, pathological examinations revealed that the ablation zone of the group B rabbits exhibited pale amorphous necrotic tissue, surrounded by cellular edema and inflammatory cell infiltration. The new capillaries were rich, and the glial cells had undergone proliferation. The pathological performance of the group A rabbits was not as typical as those in group B. Living tumor cells existed near the edema zone, with neovascularization and inflammatory cell infiltration observed among the tumor cells. The range of coagulation necrosis exhibited by pathology was consistent with that demonstrated in MRI-navigated cryoablation.

Survival analysis. Rabbits in group D exhibited appetite loss, mental malaise and lethargy, with the activity levels reducing by day 18 after the brain tumor was implanted. In addition, the rabbits gradually lost weight by day 21 , and at day 25 , the rabbits presented with symptoms of lateral limb paralysis or the body exhibited tetanic bending towards the contralateral side. Furthermore, the rabbits were restless, anxious and showed a number of signs of aggression. The average survival time of the rabbits was $32.86 \pm 3.80$ days (Fig. 2).

The seven rabbits in group $\mathrm{C}$ all successfully underwent cryoablation therapy. Following surgery, two rabbits exhibited mild hemiparesis and one rabbit had right-eye conjunctival hyperemia. Furthermore, three rabbits died on postoperative days 39, 46 and 53. The remaining rabbits were observed for 60 days to obtain censored data (Table III).

The Kaplan-Meier survival curve method was used to statistically analyze the survival times of the rabbits. The results revealed that the survival times of the rabbits in group $C$ were significantly longer compared with the rabbits in group $\mathrm{D}$, with the difference statistically significant $(\mathrm{P}<0.05)$. The survival function graph is shown in Fig. 2. 


\section{Discussion}

Establishing animal tumor models has important significance towards understanding the mechanisms underlying tumorigenesis, tumor development, tumor prevention and treatment. Rabbit VX2 cancer models of the liver, kidney, lung and bone have been studied maturely, and have been widely applied for use in tumor imaging, physiological and pathological studies, as well as in the efficacy evaluation of interventional treatment (14). However, VX2 brain tumor animal models have been rarely used in domestic and foreign studies, and the tumor implantation method used is primarily the intracerebral injection of tumor cell suspension $(15,16)$. At the start of the present study, a number of other methods had been attempted; however, various shortcomings were observed. Firstly, the concentration and amount of the injected tumor cells was not easy to control. Secondly, tumor growth was more diffuse, with the tumors growing along the needle tract. Thus, the meninges were invaded earlier, which was associated with bleeding inside the needle tract, causing the tumor cells to be flushed out. Finally, the tumor size and shape were found to differ substantially between models.

Based on the results of a previous study, the model preparation method was improved (15). Firstly, the cell suspension was replaced with a tumor block for implantation, as the block size can be more easily quantified compared with the tumor cells. Secondly, when the tumor block was implanted, a gelatin sponge was also implanted into the needle tract, which prevented the tumor block being flushed out or displaced due to the bleeding of the needle tract. Furthermore, using a gelatin sponge guaranteed that the tumor block was able to be fully implanted into the brain rather than residing inside the needle, ensuring the success of the tumor block implantation. Finally, following the implantation of the tumor block, the needle was kept in position for 3-5 min, after which it was slowly pulled out to reduce the bleeding and other complications. In total, three rabbits out of the originally prepared eight models, were randomly selected for immediate postoperative MR scanning. The results revealed that the needle tract exhibited minor bleeding; however, there were no postoperative abnormal symptoms and signs, and no rabbit died during the tumor block implantation, which demonstrated the safety of the tumor block implantation method using the puncture needle.

On postoperative day 10, normal and enhanced MRI scanning was performed in each rabbit. With the exception of one rabbit that exhibited abscess formation and presented with slight ring enhancement (reason to be discussed), the remaining rabbits exhibited tumor growth formation in the brain, with a tumor formation rate of $90.91 \%$. On postoperative day 10 , the average maximum cross-sectional diameter of the tumors was $4.4 \pm 1.1 \mathrm{~mm}$. The tumor sizes were predominantly uniform and the tumors were regularly shaped, appearing as round or oval. These observations demonstrate the successful establishment of the tumor model, which may have significance in future experimental cancer research.

In a previous study (17), with regard to the MRI scans, the tumors in the T1WI showed a slightly lower or equal signal, while the tumors in the T2WI and FLAIR scans exhibited a slightly hyperintense signal, with a clear edge and no surrounding edema. Following the injection of Gd-DTPA, the signals all showed a significant enhancement. When the tumor diameter is $>1.2 \mathrm{~cm}$, the center of the tumor may exhibit necrosis (18). The general observations of the VX2 brain tumor specimens demonstrated consistency with the imaging features, with only the volume slightly reduced compared with that in the radiographic observations. This difference may have been caused by the following two reasons. Firstly, due to the partial volume effect, the MRI scans may exhibit a slightly larger tumor diameter. Secondly, during the sampling, the $4 \%$ formaldehyde perfusion fixation may cause a reduction in the lesion volume. Tacke et al (18) considered that after fixation, the median size of the specimen was 1.14 times less compared with the measurement prior to fixation. Pathological examination revealed that cell division was exuberant, and the cells had slightly invaded towards the normal tissues. Furthermore, angiogenesis was shown to be rich. With regard to imaging observations, these pathological features primarily present as a less clear boundary and marked enhancement of the tumor.

Hypothermia is known to significantly extend the relaxation time in T1WIs, while decreasing the time in T2WIs. Thus, when performing cryoablation, the ice ball may appear as a sharp-edged hypointense signal in all MRI scanning sequences, which is conducive to the real-time display of the ice ball formation in MRI (19). According to the comparative study of pathology and MRI in the group B rabbits, the ice ball size and the tissue necrosis scope were consistent.

MRI has a temperature-sensitive characteristic, which can be used as a great advantage towards monitoring the temperature and range of the ablation zone during cryoablation of the brain tumor (12). The argon-helium knife used in the present study was a new ultra-low temperature freezing-heating therapeutic piece of equipment, which used high-pressure argon and helium as the freezing and heating medium, respectively, with the freezing principle conducted according to the Joule-Thomson effect (18). The superconductive knife was a hollow tubular probe, with the thinnest diameter of $1.47 \mathrm{~mm}$. While the knife was able to export the high-pressure argon at room temperature (the freezing medium) or helium (the heating medium), the thermocouple was mounted directly on the probe tip in order for the temperature to be continuously monitored. During the surgery, the high-pressure normal-temperature argon was firstly exported from the probe tip, causing the target tissue temperature to rapidly decrease to below $-140^{\circ} \mathrm{C}$ in 10-20 sec (argon freezing is able to reach a lowest temperature of $-185^{\circ} \mathrm{C}$ ). Subsequently, assisted by the rapid expansion of helium at the probe tip, the probe was rapidly heated to $30-50^{\circ} \mathrm{C}$ to completely destroy the tumor cells (20). Since the freezing and heating was limited to an inside range of $2 \mathrm{~cm}$ from the superconductive cryosurgical probe tip, the needle bar had good thermal insulation to prevent the occurrence of large damage to the tissues on the puncture path.

Certain experiments have confirmed that through using an intracranial glioma model, argon-helium cryoablation therapy not only causes the necrosis of glioma cells that are in the central area of the freezing zone, but also induces the apoptosis of the surrounding tumor cells (19). In addition, the in situ dead tumor tissues following cryoablation may become antigens, promoting the anti-tumor immune response of the body (18), which highlights the advantages of using argon-helium cryoablation therapy. 
MRI-navigated interventional therapy is the perfect combination of the latest imaging technology and minimally invasive surgery, possessing incomparable advantages over $\mathrm{X}$-ray, CT and B-mode ultrasound. For example, MRI utilizes multi-parameter imaging that exhibits high resolution towards the soft tissues, enabling observations of lesions that other imaging methods are unable to reveal. Furthermore, MRI has characteristics of arbitrary planar imaging and temperature-sensitive imaging. In addition, MRI is a form of functional imaging that uses safer contrast agents and produces no ionizing radiation. The open magnet ensures that the surgical procedure is more convenient. The navigation equipment and the dedicated rapid imaging sequences enable real-time guidance and monitoring throughout the operation. In addition, the flexible multi-functional coil can be placed onto any appropriate area to satisfy the surgical requests; thus, the image quality of the surgical area is improved.

Argon-helium cryoablation was performed when the rabbit VX2 brain tumor reached a diameter of $0.8 \mathrm{~cm}$ for the following reasons. Firstly, an excessively small tumor diameter is not conducive to the intraoperative identification of the tumor. Secondly, the size of the ice ball is difficult to control to within $1 \mathrm{~cm}$, and if the tumor is too small, increased damage may be caused towards the surrounding normal tissues. Finally, if the tumor is too large, the tumor-bearing rabbit can often exhibit anorexia and decreasing physical activity; therefore, tolerance towards the surgery decreases. During the process of VX2 brain tumor cryoablation, real-time dynamic scanning can be performed to observe the formation of the ice ball. The size of ice ball is largest after $4 \mathrm{~min}$, where the diameter is $1.0 \mathrm{~cm}$ and completely covers the lesion and slightly beyond the edge of the lesion. In the second cycle, the ice ball size slightly increases, reaching a diameter of $1.2 \mathrm{~cm}$. This may be the result of vascular damage or thrombosis formed in the ablation zone during the first cycle; thus, in the second cycle, the energy lost during the ablation reduces (21).

The average survival time of the rabbits in group $\mathrm{D}$ was $33 \pm 4.3$ days. When comparing the survival times between the rabbits in groups $\mathrm{C}$ and $\mathrm{D}$, a statistically significant difference $(\mathrm{P}<0.05)$ was observed. This result indicated that the argon-helium cryoablation therapy played a role in inactivating the tumor tissues, and thus, significantly improved the survival times of the tumor-bearing rabbits.

In conclusion, the present preliminary study demonstrated that the puncture needle method may be used to obtain a high success rate in the preparation of a VX2 brain tumor model. The reproducibility was good, and the tumorigenicity was uniform, safe and feasible; therefore, this method was determined to be suitable for the requirements of pathology, imaging and interventional treatment. In addition, MRI-navigated and real-time monitored argon-helium cryoablation therapy was shown to be an accurate, safe and feasible technique, with minimal invasion, for the treatment of brain tumors. The technique was found to significantly increase the survival times of the tumor-bearing rabbits, while exhibiting little effect and sequelae on the surrounding normal tissues.

\section{References}

1. Katchy KC, Alexander S, Al-Nashmi NM and Al-Ramadan A: Epidemiology of primary brain tumors in childhood and adolescence in Kuwait. Springerplus 2: 58, 2013.

2. Ziegler DS, Cohn RJ, McCowage G, et al: Efficacy of vincristine and etoposide with escalating cyclophosphamide in poor-prognosis pediatric brain tumors. Neuro Oncol 8: 53-59, 2006.

3. Lehotska V, Tothova L and Valkovic L: The role of dynamic contrast-enhanced MRI in differentiation of local recurrence and residual soft-tissue tumor versus post-treatment changes. Bratisl Lek Listy 114: 88-92, 2013.

4. Liu JG, Chen FL, Ge CL, Gong MY, Zuo HB and Zhang JR: Cryosurgery for treatment of subcutaneously xenotransplanted tumors in rats and its effect on cellular immunity. Technol Cancer Res Treat 10: 339-346, 2011.

5. Yang Y, Wang C, Lu Y, et al: Outcomes of ultrasound-guided percutaneous argon-helium cryoablation of hepatocellular carcinoma. J Hepatobiliary Pancreat Sci 19: 674-684, 2012.

6. Vasil'ev SA, Pesnia-Prasolov SB, Krylov VV, et al: Cryodestruction in neurosurgery. Khirurgiia (Mosk) 2: 105-108, 2013 (In Russian).

7. Bomers JG, Yakar D, Overduin CG, et al: MR imaging-guided focal cryoablation in patients with recurrent prostate cancer. Radiology 268: 451-460, 2013.

8. Jaskólski DJ, Fortuniak J, Majos A, et al: Magnetic resonance spectroscopy in intracranial tumours of glial origin. Neurol Neurochir Pol 47: 438-449, 2013.

9. Sun CJ, Li C, Yu JM, et al: Comparison of 64-slice CT perfusion imaging with contrast-enhanced CT for evaluating the target volume for three-dimensional conformal radiotherapy in the rabbit VX2 brain tumor model. J Radiat Res 53: 454-461, 2012.

10. Bahn D, de Castro Abreu AL, Gill IS, et al: Focal cryotherapy for clinically unilateral low-intermediate risk prostate cancer in 73 men with a median follow-up of 3.7 years. Eur Urol 62: 55-63, 2012.

11. Gangi A, Tsoumakidou G, Abdelli O, et al: Percutaneous MR-guided cryoablation of prostate cancer: initial experience. Eur Radiol 22: 1829-1835, 2012.

12. Anayama T, Nakajima T, Dunne M, et al: A novel minimally invasive technique to create a rabbit vX2 lung tumor model for nano-sized image contrast and interventional studies. PLoS One 8: e67355, 2013.

13. He Y, Tu HJ, Lei Z, et al: Experimental study of apoptosis in C6 rat glioma model induced by cryotherapy. Zhonghua Zhong Liu Fang Zhi Za Zhi 12: 1615-1618, 2005 (In Chinese).

14. Song J, Li C, Wu L, et al: MRI-guided brain tumor cryoablation in a rabbit model. J Magn Reson Imaging 29: 545-551, 2009.

15. Mei LJ, Yang XJ, Tang L, Hassan AH, Yonemura Y and Li Y: Establishment and identification of a rabbit model of peritoneal carcinomatosis from gastric cancer. BMC Cancer 10: 124, 2010.

16. Handal JA, Schulz JF, Florez GB, Kwok SC, Khurana JS and Samuel SP: Creation of rabbit bone and soft tissue tumor using cultured VX2 cells. J Surg Res 179: e127-e132, 2013.

17. Anderson JK, Shingleton WB and Cadeddu JA: Imaging associated with percutaneous and intraoperative management of renal tumors. Urol Clin North Am 33: 339-352, 2006.

18. Tacke J, Speetzen R, Adam G, et al: Experimental MR imaging-guided interstitial cryotherapy of the brain. AJNR Am J Neuroradiol 22: 431-440, 2001.

19. Hu KW, Li QW, Zuo MH, Sun T and Jiang M: Clinical observation on the combined treatment of 57 cases of non-small cell lung cancer using argon-helium cryosurgery and Chinese herbal medicine. Chin J Integr Med 13: 224-227, 2007.

20. Mack MG and Vogl TJ: MR-guided ablation of head and neck tumors. Neuroimaging Clin N Am 14: 853-859, 2004.

21. Xiao H, Liao Q, Cheng M, et al: 5-Amino-4-oxopentanoic acid photodynamic diagnosis guided microsurgery and photodynamic therapy on VX2 brain tumour implanted in a rabbit model. Chin Med J (Engl) 122: 1316-1321, 2009. 